\section{Pharmaceutical progress}

\section{S.S. Davis}

Anti-Cancer Drug Design. Editors Stanley Crooke, Stephen Neidle and Shigeru Tsukagoshi. Macmillan, London. 4/yr. $U K £ 55$; elsewhere $£ 75$.

Drug Design and Delivery. Editor J.S Morley. Harwood. 4 issues per volume. $\$ 286$ (institutional); $\$ 194$ (libraries).

THE greater understanding of the biology of cancer at the molecular level has meant that it is possible to take a more rational approach to the design of anti-cancer agents. Such an approach employs the tools of modern molecular and structural biology as well as the more traditional methods of pharmacology, medicinal chemistry and biophysics.

Anti-Cancer Drug Design has been introduced to provide a unifying forum for these developments. The articles in the issues available for review are wide ranging in scope. Some of them present details of conventional medicinal chemistry, with descriptions of the synthesis of new analogues and their in vitro and in vivo assessment, while others consider aspects related to the effective delivery of anti-cancer agents to include antibodytargeted drug-carrier conjugates and drug-loaded microparticulates.

So far the journal appears to be achieving its objective of publishing research papers that involve the application of a rationality of approach rather than the mere listing of compounds synthesized and tested. Clinically orientated material, although welcomed, has yet to make a strong showing. The papers are in the form of conventional articles as well as short communications, and are generally of high quality. One 'mini' review has appeared so far; this was most informative and I hope that further such reviews will appear in forthcoming issues. The journal is well produced and so far the papers have been published rapidly after acceptance.

As with any new venture, it is vital that the initial enthusiasm generated by the editors does not wane and that potential authors do not revert to their previous outlets for publication. The field of cancer research is well served by a variety of journals and the same is true of medicinal chemistry; indeed, another journal that cuts across some of the material has also been introduced recently (Cancer Drug Delivery, published by Mary Ann Liebert Inc.). Today, the survival of any new journal is doubtful unless it fills an obvious gap. Anti-Cancer Drug Design, published with support from the Cancer Research Campaign, has a better chance than most.

In justifying Drug Design and Delivery,
Being industrious

the other journal reviewed here, the not catered for in the existing scientific press". Once again, the rationale is to provide a multi-disciplinary setting for concepts arising from chemistry, biochemistry, pharmacology, immunology and even mathematics. In doing so, it is hoped to create a comprehensive source of current information on innovative approaches to drug design, absorption, distribution, metabolism, release and finally clinical effect.

Whether such a gap actually exists is debatable. Journal of Pharmaceutical Sciences and Pharmaceutical Research could be said to have exactly the same intentions. Interestingly, it is hoped that the editorial policy will attract papers from individual scientists in the pharmaceutical industry who have been reluctant to publish in the past. It is certainly true that the publication policies of many pharmaceutical companies are overcautious, but how the present journal would alter the situation is not explained.

The papers that have appeared to date

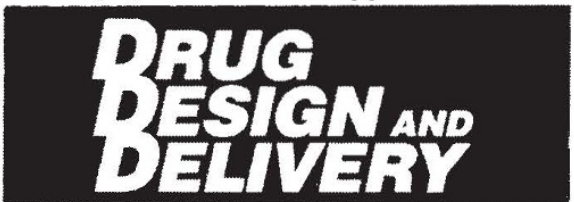

have been of mixed quality, and have dealt either with drug design or with delivery. Few have yet combined the two. The standard of presentation is generally high, and it is good to note that colour, when an integral part of the research, is published without charge to the authors. Papers are either typeset or, for rapid publication, are in a camera-ready form.

The one book review that has appeared so far (in the first issue of the journal) considered the book entitled Multi-dimensional Pharmacochemistry by P.P. Mager. The reviewers were not overly impressed by what they read. Most unusually, the author takes the reviewers to task in a letter to the editor in a subsequent issue. These two contributions make far more interesting reading than many of the scientific contributions.

Only time will tell whether this journal is able to establish a place for itself within the pharmaceutical sciences. Better quality papers need to be attracted, and the editorial board could play a role here. Three of its members are scientists who have had prestigious careers in the pharmaceutical industry and have now moved into academia; perhaps they could encourage their former colleagues to publish more! One member of the board has certainly been of considerable benefit of the 35 papers published so far, he is the co-author of ten.

S.S Davis is Lord Trent Professor of Pharmacy at the University of Nottingham, University Park, Nottingham NG72RD, UK.

\section{Colin Webb}

Bioprocess Engineering. Managing editor H. Brauer. Springer-Verlag. 4/yr. DM 196 plus carriage charge.

THERE are currently more than 250 periodicals world-wide which serve the broad field of biotechnology, with new titles emerging by the month. Remarkably, for a technology with such commercial potential, very few of these deal with process engineering. Indeed, apart from The Biochemical Engineering Journal, which is published jointly with The Chemical Engineering Journal, and Advances in Biochemical Engineering, which is published annually, there are no English-language periodicals dedicated entirely to such matters. Bioprocess Engineering therefore fills an important gap in the literature. It publishes original papers, short communications and occasional review articles, on "all technical and economical aspects of processes in which natural or derived biological substances are the basic material".

In line with most quality journals, Bioprocess Engineering is produced in a twocolumn, A4 format, with all the text being typeset and figures being reproduced directly from the authors' originals. Proof reading of articles is the responsibility of the individual authors, which is just as well because the journal's own proof readers are guilty of fairly serious errors, from time to time, in the contents pages and index lists. Happily, pre-publication delays are not apparent, and most contributions appear within six to nine months of receipt of the manuscript.

The technical content is generally of a high standard, with most contributions keeping within the intended scope of the journal. Although, in its first year, 30 per cent of the papers came from the editors or their advisory board, none appeared to be merely page fillers and all presented useful new design information. As any industrial technology comes of age there becomes an increasing need for sound engineering evaluations of available information. Articles such as that by Schumpe and Deckwer (Vol. 2, pp. 7994) fulfil such a need by not only supplying valuable new information but also by critically reviewing, and correlating, existing information from the literature. Such contributions, along with the many others of similar quality, make Bioprocess Engineering an invaluable addition to any biotechnology or process engineering library.

Colin Webb is a Lecturer in the Department of Chemical Engineering, University of Manchester Institute of Science and Technology, PO Box 88, Manchester M60 1QD, UK. 